Japan. J. Med. Sci. Biol., 27, 277-284, 1974

\title{
GEOGRAPHICAL DISTRIBUTION OF LEPTOSPIRA-POSITIVE FIELD RODENTS IN JAPAN
}

Many papers have been published on leptospira-positive rodents in selected areas of Japan. Rattus norvegicus, $R$. rattus, and $R$. rattus alexandrius trapped in urban areas and certain swamp rice fields in rural areas were found to carry L. icterohaemorrhagiae (Ido et al., 1916; Higuchi, 1930; Kitaoka, Inoue and Kubo, 1935). Microtus montebelli and Apodemus speciosus speciosus were reported to carry L. hebdomadis (Ido, Ito and Wani, 1917) and L. autumnalis (Koshina, Shiozawa and Kitayama, 1924; Abe et al., 1933; Kitaoka and Ogawa, 1942), respectively. The latter two species of rodents are caught generally in the field and forest in rural areas. Up to the present, at least nine serotypes of pathogenic leptospira, L. icterohaemorrhagiae, L. hebdomadis, L. autumnalis, L. australis (Shiozawa and Kubo, 1938), L. canicola (Kitaoka, 1940; Yamamoto, 1940), L. bataviae (Kitaoka, 1951), L. pyrogenes (Yamamoto and Hashiguchi, 1944; Kitaoka, Maida and Inoue, 1950), L. javanica (Kobayashi, Kusaba and Ueki, 1972) and L. grippotyphosa (Kitaoka et al., unpublished data), have been isolated from the rodent in Japan. With field rodents and other animals trapped in a country-wide survey on rickettsia-positive animals (Tamiya, 1962), attempts have been made to know what species of animals are leptospira positive and to elucidate geographical distribution of such animals in Japan excluding the Okinawa Islands.

Of all animals trapped in 428 areas in Japan during the period 1955-1962, 4,108 were used in this investigation. The animals were identified finally by Dr. Y. Imaizumi, the National Science Museum (Natural History Institute), Tokyo. They were comprised of 35 species and their geographical distribution is given in Table I and was illustrated in more details in a book (Tamiya, 1962). The kidneys to be tested were fixed in $5 \%$ formalin and stained by Levaditi method. The section of the stained kidney was examined under a microscope; leptospires were morphologically confirmed in the lumen of the convoluted tubules of the kidney (Fig. 1).

Figure 2 indicates geographical distribution of leptospira-positive and -negative animals in 428 areas in 38 prefectures from Wakkanai, the northernmost town in Hokkaido, to Amami Oshima Island, the southernmost island in Kagoshima prefecture. In some areas repeated surveys were performed in different seasons, but generally a single survey of an area was conducted during a certain season. Our former surveys (Kitaoka et al., 1935; Kitaoka and Ogawa, 1942) on leptospira-positive field rodents revealed that the heavier the body weights of rodents, the higher the leptospirapositive rate in the kidneys. Accordingly, negative areas on the map do not strictly mean no-epidemicity in leptospira-positive small animals. One hundred and twentyfive of 428 areas surveyed were found to be leptospira-positive. These areas included Shiretoko Peninsula, Hokkaido in the north, and Amami Oshima Island, Kagoshima prefecture in the south.

Table II indicates the species of field rodents and other small animals trapped for leptospira surveillance in eight districts in Japan. One hundred and forty-six of 


\section{TABLE I}

Leptospira-positive rates of small animals trapped in Japan, 1955-1962

\begin{tabular}{|c|c|c|c|c|c|c|c|c|c|c|}
\hline \multirow{2}{*}{$\begin{array}{c}\text { Small } \\
\text { animal }\end{array}$} & \multicolumn{10}{|c|}{ District } \\
\hline & $\begin{array}{l}\text { Hok- } \\
\text { kaido }\end{array}$ & Tohoku & Kanto & Chubu & Kinki & $\begin{array}{l}\text { Chu- } \\
\text { goku }\end{array}$ & $\begin{array}{l}\text { Shi- } \\
\text { koku }\end{array}$ & $\begin{array}{c}\text { Kyu- } \\
\text { shu }\end{array}$ & Total & $\begin{array}{c}\text { Per } \\
\text { cent }\end{array}$ \\
\hline A.a.a. & $0 / 11$ & $2 / 50$ & $0 / 41$ & $1 / 83$ & $0 / 12$ & $0 / 22$ & $0 / 3$ & $0 / 2$ & $3 / 224$ & 1.3 \\
\hline A.a.c. & & & & & & $0 / 11$ & & & $0 / 11$ & 0 \\
\hline A.a.h. & $1 / 56$ & & & & & & & & $1 / 56$ & 1.8 \\
\hline A.a.s. & & & & & & & & $0 / 10$ & $0 / 10$ & 0 \\
\hline A.a.y. & & & & & & & & $0 / 6$ & $0 / 6$ & 0 \\
\hline A.s.a. & $2 / 52$ & & & & & & & & $2 / 52$ & 3.8 \\
\hline A.s.d. & & & & & & & & $0 / 48$ & $0 / 48$ & 0 \\
\hline A.s.i. & & & $0 / 33$ & & & & & & $0 / 33$ & 0 \\
\hline A.s.n. & & & & & & $2 / 87$ & & & $2 / 87$ & 2.3 \\
\hline A.s.s. & & $4 / 147$ & $6 / 340$ & $35 / 503$ & $0 / 92$ & $19 / 388$ & $2 / 143$ & $17 / 182$ & $83 / 1795$ & 4.6 \\
\hline A.s.sa. & & & & $0 / 10$ & & & & & $0 / 10$ & 0 \\
\hline A.s.t. & & & & & & & & $0 / 1$ & $0 / 1$ & $*$ \\
\hline As.a. & & $0 / 2$ & & $0 / 3$ & & & $0 / 2$ & & $0 / 7$ & 0 \\
\hline C.d.d. & & & & & & & $0 / 1$ & & $0 / 1$ & $*$ \\
\hline C.r.b. & $7 / 366$ & & & & & & & & $7 / 366$ & 1.9 \\
\hline C.r.m. & $0 / 1$ & & & & & & & & $0 / 1$ & * \\
\hline E.k. & & $0 / 1$ & $2 / 8$ & $4 / 120$ & & & & & $6 / 129$ & 4.7 \\
\hline E.s.o. & & & & & & $0 / 13$ & & & $0 / 13$ & 0 \\
\hline E.s.s: & & & & & $0 / 10$ & $0 / 23$ & $0 / 3$ & & $0 / 36$ & 0 \\
\hline G.j. & & $0 / 6$ & & & & & & & $0 / 6$ & 0 \\
\hline M.c.c. & & & & & & & & $1 / 1$ & $1 / 1$ & $*$ \\
\hline M.m: & & $0 / 167$ & $0 / 8$ & $22 / 458$ & $0 / 2$ & $0 / 9$ & & & $22 / 644$ & 3.4 \\
\hline M.m.m. & & & & $0 / 1$ & & & & & $0 / 1$ & * \\
\hline M.m.o. & & & & & & & & $0 / 1$ & $0 / 1$ & $*$ \\
\hline R.n. & $2 / 14$ & $5 / 15$ & $5 / 97$ & $3 / 42$ & & $0 / 3$ & $2 / 59$ & $0 / 57$ & $17 / 287$ & 5.9 \\
\hline R.r. & & $0 / 8$ & $0 / 85$ & $0 / 4$ & & & $0 / 39$ & $1 / 43$ & $1 / 179$ & 0.6 \\
\hline S.m.r. & & & & & & & & $0 / 7$ & $0 / 7$ & 0 \\
\hline S.s.sa. & $0 / 4$ & & & & & & & & $0 / 4$ & 0 \\
\hline S.s.sh & & $0 / 2$ & & & & & & & $0 / 2$ & $*$ \\
\hline S.u. & $0 / 7$ & & & & & & & & $0 / 7$ & 0 \\
\hline U.t.c. & & & & & & & $0 / 7$ & & $0 / 7$ & 0 \\
\hline U.t.h. & & $0 / 7$ & $0 / 2$ & $0 / 14$ & $0 / 16$ & $0 / 4$ & & & $0 / 43$ & 0 \\
\hline U.t.m. & & & & & & $0 / 4$ & & & $0 / 4$ & 0 \\
\hline U.t.t. & & & & & & & & $1 / 7$ & $1 / 7$ & 14.3 \\
\hline To.o.o: & & & & & & & & $0 / 18$ & $0 / 18$ & 0 \\
\hline Total & $12 / 511$ & $11 / 405$ & $13 / 614$ & $65 / 1238$ & $0 / 132$ & $23 / 562$ & $4 / 257$ & $20 / 383$ & $146 / 4104$ & 3.6 \\
\hline Percent & 2.3 & 2.7 & 2.1 & 5.3 & 0 & 3.7 & 1.6 & 5.2 & 3.6 & \\
\hline
\end{tabular}


A.a.a.: Apodemus argenteus argenteus

A.a.c.: Apodemus argenteus celatus

A.a.h.: Apodemus argenteus hokkaidi

A.a.s.: Apodemus argenteus sagax

A.a.y.: Apodemus argenteus yakui

A.s.a.: Apodemus speciosus ainu

A.s.d.: Apodemus speciosus dorsalis

A.s.i.: Apodemus speciosus insperatus

A.s.n.: Apodemus speciosus navigator

A.s.s.: Apodemus speciosus speciosus

A.s.sa.: Apodemus speciosus sadoensis

A.s.t.: Apodemus speciosus tusimaensis

As.a.: Aschigomys andersoni

C.d.d.: Crochidura desi-nezumi desi-nezumi

C.r.b.: Clethrionomys rufocanus bedfordiae

C.r.m.: Clethrionomys rutilus mikado

E.k.: Eothenomys kageus

E.s.o.: Eothenomys smithi okiensis
E.s.s.: Eothenomys smithi smithi

G.j.: $\quad$ Glirulus japonicus

M.c.c.: Mus caroli caroli

M.m.: Microtus montebelli

M.m.m.: Mus musculus molossinus

M.m.o.: Mus musculus orii

R.n.: Rattus norvegicus

R.r.: Rattus rattus

S.m.r.: Suncus murinus riukiuanus

S.s.sa.: Sorex shinto saevus

S.s.sh.: Sorex shinto shinto

S.u.: Sorex unguiculatus

U.t.c.: Urotrichus talpoides centralis

U.t.h.: Urotrichus talpoides hondonis

U.t.m.: Urotrichus talpoides minutus

U.t.t.: Urotrichus talpoides talpoides

To.o.o.: Tokudaia osimensis osimensis

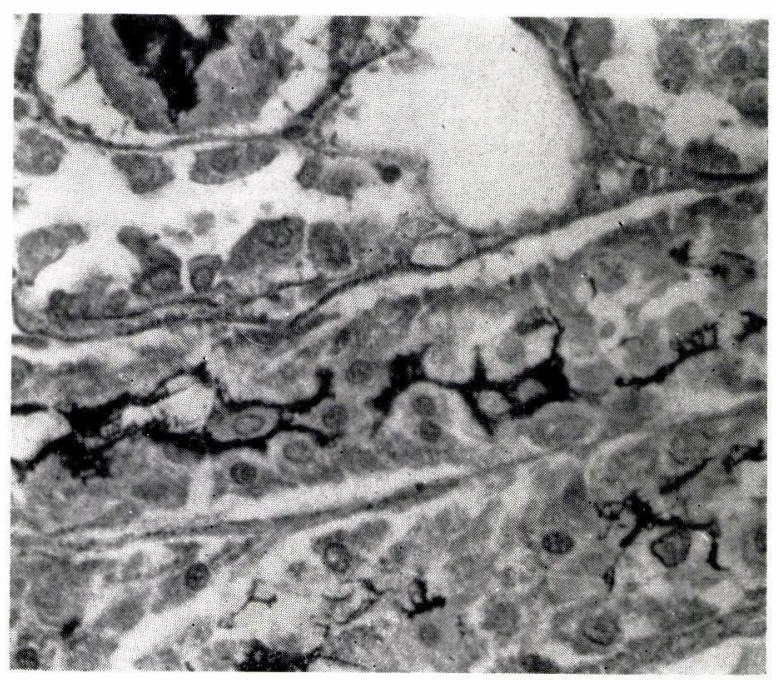

Fig. 1. Leptospires in the lumen of the convoluted tubules of the kidney of Apodemus speciosus speciosus, male, body weight $50 \mathrm{~g}$, trapped in Nobeoka, Miyazaki prefecture, Kyushu on August 17, 1957. Stained by Levaditi method (×600).

4,104 animals tested comprising 35 species were leptospira-positive. There were the following 12 species: Apodemus argenteus argenteus, A. argenteus hokkaidi, $A$. speciosus ainu, A. speciosus navigator, A. speciosus speciosus*, Clethrionomys rufocanus bedfordiae, Eothenomys kageus, Mus caroli caroli, Microtus montebelli*, Rattus norvegicus*, Rattus ruttus*, and Urotrichus talpoides talpoides. Four species marked with asterisks had been found to be leptospira-positive. The remaining eight species were found for the first time to be positive. The average positive rate was $3.6 \%$. The rates for individual species of rodents were $5.9 \%$ for $R$. norvegicus, $4.6 \%$ for A. speciosus, $3.8 \%$ for A. speciosus ainu, and $3.4 \%$ for $M$. montebelli $(U$. talpoides talpoides was positive at $14.3 \%$ in an examination). It can be said that the more positive spots located in one area, the higher the incidence of leptospirosis cases, for example, in Niigata, 
TABLE II

Monthly fuctuation of leptospira-positive rates in small animals trapped in various areas in Japan, 1955-1962

\begin{tabular}{|c|c|c|c|c|c|c|c|}
\hline \multirow{2}{*}{$\begin{array}{l}\text { Small } \\
\text { animal }\end{array}$} & \multicolumn{7}{|c|}{ Month } \\
\hline & Jan. & Feb. & Mar. & Apr. & May & Jun. & Jul. \\
\hline A.a:a. & $0 / 10$ & $1 / 19$ & $0 / 6$ & $0 / 5$ & $0 / 31$ & $2 / 46$ & $0 / 19$ \\
\hline A.a.c. & $0 / 1$ & & $0 / 4$ & & & $0 / 4$ & \\
\hline $\begin{array}{l}\text { A.a.h. } \\
\text { A.a.s. }\end{array}$ & & $0 / 4$ & $0 / 1$ & & $0 / 2$ & $1 / 25$ & $0 / 3$ \\
\hline A.a.y. & & $0 / 6$ & & & & & \\
\hline A.s.a. & & & & & & $2 / 19$ & $0 / 3$ \\
\hline $\begin{array}{l}\text { A.s.d. } \\
\text { A.s.i. }\end{array}$ & & $0 / 48$ & & & & & \\
\hline A.s.n. & $0 / 9$ & & $0 / 18$ & & & $0 / 26$ & \\
\hline $\begin{array}{l}\text { A.s.s. } \\
\text { A.s.sa. }\end{array}$ & $0 / 85$ & $6 / 214$ & $14 / 281$ & $0 / 111$ & $1 / 148$ & $9 / 120$ & $3 / 149$ \\
\hline A.s.t. & $0 / 1$ & & & & & & \\
\hline $\begin{array}{l}\text { As.a. } \\
\text { C.d.d. }\end{array}$ & & $0 / 1$ & & & & & $0 / 2$ \\
\hline $\begin{array}{l}\text { C.r.b. } \\
\text { C.r.m. }\end{array}$ & $0 / 20$ & $0 / 25$ & $0 / 26$ & $0 / 23$ & $0 / 20$ & $5 / 91$ & $0 / 8$ \\
\hline $\begin{array}{l}\text { E.k. } \\
\text { E.s.o. }\end{array}$ & $0 / 12$ & $2 / 11$ & $\begin{array}{l}0 / 4 \\
0 / 10\end{array}$ & $0 / 3$ & $0 / 12$ & $\begin{array}{l}0 / 13 \\
0 / 3\end{array}$ & $0 / 3$ \\
\hline E.s.s. & & $0 / 9$ & $0 / 1$ & $0 / 8$ & & & \\
\hline $\begin{array}{l}\text { G.j. } \\
\text { M.c.c. }\end{array}$ & & & & & & $0 / 6$ & \\
\hline $\begin{array}{l}\text { M.m. } \\
\text { M.m.m. }\end{array}$ & $0 / 17$ & $3 / 16$ & $0 / 28$ & $1 / 60$ & $0 / 60$ & $0 / 98$ & $0 / 33$ \\
\hline M.m.o. & & & & & & & $0 / 1$ \\
\hline R.n. & $0 / 15$ & $0 / 2$ & $0 / 5$ & $0 / 7$ & $0 / 6$ & $1 / 25$ & $2 / 41$ \\
\hline $\begin{array}{l}\text { R.r. } \\
\text { S.m.r. }\end{array}$ & $1 / 38$ & $0 / 9$ & $0 / 2$ & $0 / 2$ & & & $0 / 20$ \\
\hline S.s.sa. & & & $0 / 3$ & & & & \\
\hline S.s.sh. & & & & $0 / 2$ & & & \\
\hline S.u. & & $0 / 6$ & & & & & \\
\hline U.t.c. & & $0 / 4$ & & & & & \\
\hline U.t.h. & $0 / 1$ & $0 / 20$ & $0 / 2$ & $0 / 2$ & $0 / 6$ & & \\
\hline U.t.m. & & & $0 / 2$ & & & & \\
\hline $\begin{array}{l}\text { U.t.t. } \\
\text { To.o.o. }\end{array}$ & & $0 / 4$ & & & & & $0 / 1$ \\
\hline Total & $1 / 209$ & $13 / 398$ & $14 / 393$ & $1 / 223$ & $1 / 285$ & $20 / 276$ & $5 / 283$ \\
\hline Percent & 0.5 & 3.3 & 3.6 & 0.4 & 0.4 & 4.2 & 1.8 \\
\hline
\end{tabular}




\begin{tabular}{|c|c|c|c|c|c|c|}
\hline \multicolumn{5}{|c|}{ Month } & \multirow{2}{*}{ Total } & \multirow{2}{*}{$\begin{array}{c}\text { Per } \\
\text { cent }\end{array}$} \\
\hline Aug. & Sep. & Oct. & Nov & Dec. & & \\
\hline \multirow[t]{2}{*}{$0 / 6$} & $0 / 46$ & $0 / 10$ & $0 / 12$ & $0 / 14$ & $3 / 224$ & 1.3 \\
\hline & $0 / 2$ & & & & $0 / 11$ & 0 \\
\hline \multirow[t]{3}{*}{$0 / 13$} & $0 / 7$ & & $0 / 1$ & & $1 / 56$ & 3.6 \\
\hline & & $0 / 10$ & & & $0 / 10$ & 0 \\
\hline & & & & & $0 / 6$ & 0 \\
\hline \multirow[t]{2}{*}{$0 / 16$} & $0 / 12$ & & $0 / 2$ & & $2 / 52$ & 3.8 \\
\hline & & & & & $0 / 48$ & 0 \\
\hline \multirow[t]{2}{*}{$0 / 2$} & & & $0 / 15$ & $0 / 16$ & $0 / 33$ & 0 \\
\hline & $2 / 34$ & & & & $2 / 87$ & 2.3 \\
\hline \multirow[t]{5}{*}{$7 / 69$} & $10 / 186$ & $11 / 168$ & $1 / 157$ & $21 / 107$ & $83 / 1795$ & 4.6 \\
\hline & & $0 / 4$ & $0 / 6$ & & $0 / 10$ & 0 \\
\hline & & & & & $0 / 1$ & $*$ \\
\hline & & $0 / 2$ & $0 / 3$ & & $0 / 7$ & 0 \\
\hline & & & & & $0 / 1$ & $*$ \\
\hline $0 / 72$ & $2 / 60$ & $0 / 11$ & $0 / 10$ & & $7 / 366$ & 1.9 \\
\hline $0 / 1$ & & & & & $0 / 1$ & $*$ \\
\hline \multirow[t]{4}{*}{$0 / 2$} & $1 / 14$ & $2 / 30$ & $1 / 11$ & $0 / 14$ & $6 / 129$ & 4.7 \\
\hline & & & & & $0 / 13$ & 0 \\
\hline & $0 / 18$ & & & & $0 / 36$ & 0 \\
\hline & & & & & $0 / 6$ & 0 \\
\hline $1 / 1$ & & & & & $1 / 1$ & * \\
\hline \multirow[t]{3}{*}{$1 / 145$} & $5 / 29$ & $3 / 32$ & $2 / 65$ & $6 / 61$ & $21 / 644$ & 3.4 \\
\hline & $0 / 1$ & & & & $0 / 1$ & $*$ \\
\hline & & & & & $0 / 1$ & $*$ \\
\hline $2 / 81$ & $0 / 4$ & $1 / 30$ & $0 / 15$ & $11 / 56$ & $17 / 287$ & 5.9 \\
\hline $0 / 32$ & $0 / 5$ & $0 / 16$ & $0 / 37$ & $0 / 18$ & $1 / 179$ & 0.6 \\
\hline \multirow[t]{9}{*}{$0 / 7$} & & & & & $0 / 7$ & 0 \\
\hline & & & $0 / 1$ & & $0 / 4$ & 0 \\
\hline & & & & & $0 / 2$ & $*$ \\
\hline & $0 / 1$ & & & & $0 / 7$ & 0 \\
\hline & $0 / 3$ & & & & $0 / 7$ & 0 \\
\hline & $0 / 1$ & $0 / 5$ & $0 / 5$ & $0 / 1$ & $0 / 43$ & 0 \\
\hline & $0 / 2$ & & & & $0 / 4$ & 0 \\
\hline & & & & $1 / 2$ & $1 / 7$ & 14.3 \\
\hline & & & & $0 / 18$ & $0 / 18$ & 0 \\
\hline $11 / 447$ & $20 / 425$ & $17 / 310$ & $4 / 340$ & $39 / 307$ & $146 / 4104$ & 3.6 \\
\hline 2.5 & 4.7 & 5.3 & 1.2 & 12.7 & 3.6 & \\
\hline
\end{tabular}




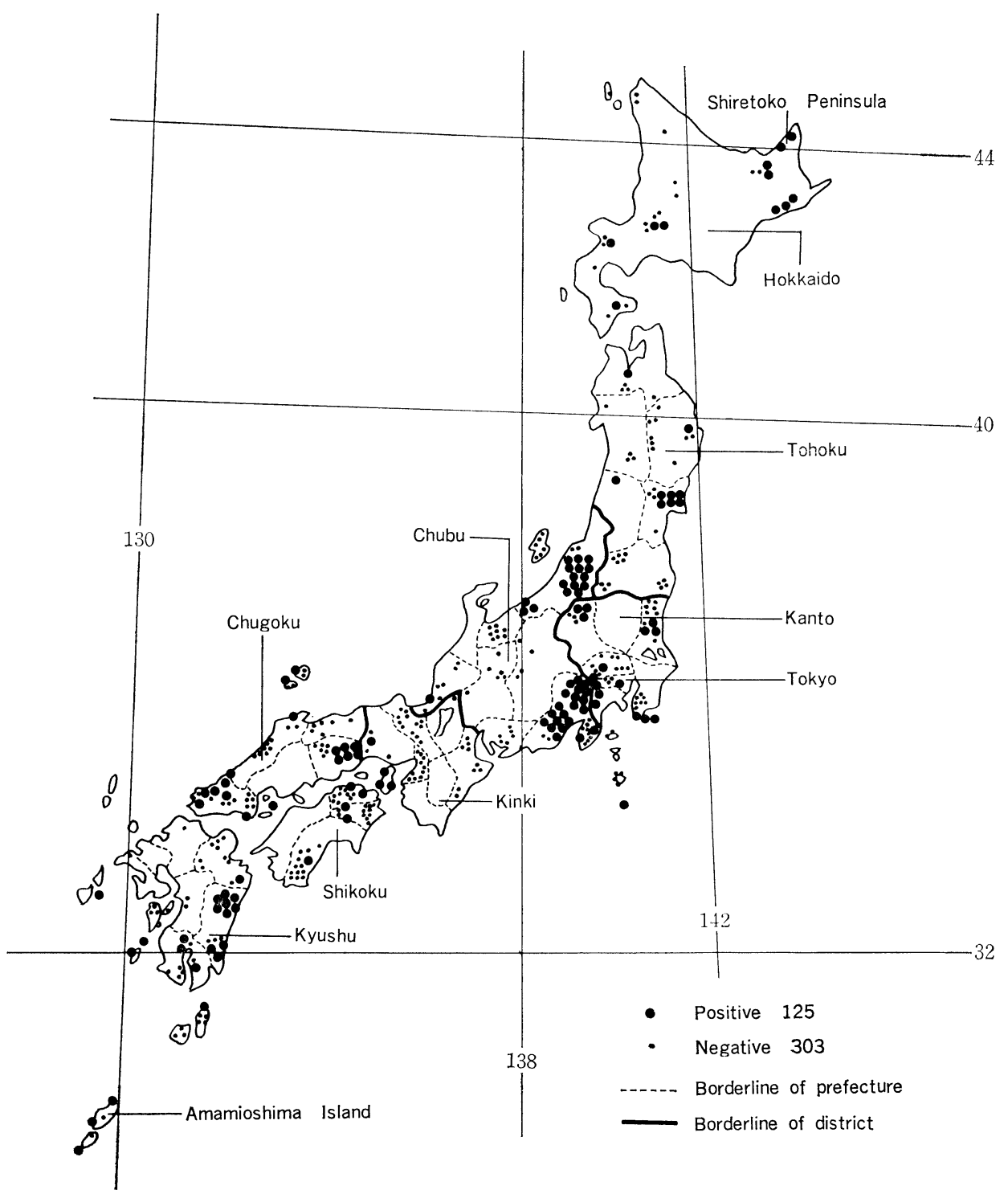

Fig. 2. Geographical distribution of leptospira-positive small animals all over Japan, 19551962. A similar figure appears on page 212 "Vaccines in Japan", Maruzen Co. Ltd., Tokyo, 1962 (in Japanese).

Miyagi, Ibaraki, Shizuoka and some other areas.

Table II shows the relation between the positive rate and the month at which time the small animals were trapped. The positive rate ranged from 1.2 to $12.7 \%$ in a period from February through March and from June through December.

Investigation of serotypes of leptospira in leptospiral animals and identification of leptospiral isolates from them should be made next, since classification and identi- 
fication of serotypes, known and unknown, of leptospira found in animals, especially in the newly discovered eight species of leptospiral animals are practically important to study epidemiology of leptospirosis and its control. In addition, those eight species (A. argenteus argenteus, A. argenteus hokkaidi, A. speciosus ainu, A. speciosus navigator, Clethrionomys rufocanus bedfordiae, Eothenomys kageus, Mus caroli caroli, and Urotrichus talpoides talpoides) are to be added to the lists of leptospiral host animals (US Department of Public Health, 1966).

\section{ACKNOWLEDGEMENT}

The authors wish to thank all members of the Group on the Scrub Typhus Study for their trapping animals all over Japan. The Group's study was partly supported by a grant-in-aid (contract No. DA-92-557-FEC-38304) of U.S. Government.

\section{REFERENCES}

Abe, T., Tokunaga, T., Kaneko, M., Kotorit, S. And Aoki, G. (1933): On natural reservoir of Hasaminetsu. Causative agent in the Hasami area, Nagasaki Prefecture. Nihon Densenbyo Gakkai Zasshi, 7, 565-578 (in Japanese).

Higuchi, S. (1930): Positive rates of Leptospira icterohaemorrhagiae in house rats trapped at various areas in Japan. Tokyo Iji-shinshi (No. 2704) 2722-2727, and (No. 2705) 27972802 (in Japanese).

IDO, Y., HoKI, R., ITO, H. AND WANI, H. (1916): House rats as a carrier of Leptospira icterohaemorrhagiae. Relationship between rats and mode of infection. Tokyo Iji-Shinshi (No. 1978), 1387-1397, and (No. 1979), 1439-1448 (in Japanese).

IDO, Y., ITO, H. AND WANI, H. (1917): Relationship between field rodents and Nanukayami. Nihon Naikagaku Zasshi, 5, 274-278 (in Japanese).

KITAOKA, M. (1940): Leptospira canicola. Jikken Igaku Zasshi, 24, 861 (in Japanese).

KITAOKA, M. (1951): Classification of leptospira. Japan. Med. J., 4, 181-214.

KitAOKA, M., INOUE, K. AND KUBO, I. (1935): Leptospira icterohaemorrhagiae in house rats in Tokyo. Tokyo Igakkai Zasshi, 9, 851-862 (in Japanese).

Kitaoka, M. AND OGAWA, M. (1942): Positive rates of leptospira in field rodents trapped at the Akiyami endemic area in Shizuoka Prefecture. Nihon Igaku Kenkohoken, (No. 3310), 2498-2503 (in Japanese).

KITAOKA, M., MAIDA, D. AND INOUE, Y. (1950): A febrile disease in Kumamoto following the typhoon July, 1949. Igaku Tsushin, (No. 221), 4-6 (in Japanese).

KobAYASHI, Y., KUSABA, T. AND UEKI, R. (1972): Isolation of $L$. javanica from rats on Ishigaki Island. Am. J. Trop. Med. Hyg., 21, 342-344.

Koshina, Y., ShiozawA, S. AND KitaYAMA, K. (1924): Relationship between field rodents and Akiyami leptospira. Iji Shimbun, (No. 1140), 581-587 (in Japanese).

SHIOZAWA, S. AND KUBO, I. (1938): Isolation of a new leptospira from a case of "Akiyami fever" (so-called Yosui disease) in the area along the Tenryu River in Shizuoka (1). Tokyo Iji-Shinshi, (No. 3097), 2199-2203 (in Japanese).

TAMIYA, T. [ed.] (1962): Recent advances in studies of tsutsugamushi disease in Japan. Medical Culture Inc., Tokyo, $309 \mathrm{p}$.

U.S. Department of Health, Education and Welfare (1966): Leptospiral Serotype Distribution Lists. U.S. Government Printing Office, Washington, D. C. 66 p.

Yамамото, S. (1940): Leptospirosis in dogs (1). Jap. J. Vet. Sci., 2, 39-41 (in Japanese).

YAMAMOTo, S. AND HASHIGUCHI, W. (1944): Leptospira pyrogenes (Vervoort 1926) in Okinawa. Nihon Igaku Kenkohoken, (No. 3333) (in Japanese). 
Department of Virology and Rickettsiology

MASAMI KITAOKA*

National Institute of Health,

MASAKO ISHIMARU**

Shinagawa-ku, Tokyo 141, Japan

(Received: May 8, 1974)

** The 2nd Department of Bacteriology, National Institute of Health.

北岡正見・石丸鉦子（国立予防衛生研究所 ウイルス・リケッチャ部） 